Authors

Xinye Xu, Thomas H. Loftus, Matthew J. Smith, John L. Hall, Alan Gallagher, and Jun Ye 


\title{
Dynamics in a two-level atom magneto-optical trap
}

\author{
Xinye Xu, ${ }^{*}$ Thomas H. Loftus, Matthew J. Smith, John L. Hall, Alan Gallagher, and Jun Ye ${ }^{\dagger}$ \\ JILA, National Institute of Standards and Technology and University of Colorado, Boulder, Colorado 80309-0440
}

(Received 20 March 2002; published 15 July 2002)

\begin{abstract}
Alkaline-earth-metal atoms present an ideal platform for exploring magneto-optic trap (MOT) dynamics, enabling unique and definitive tests of laser cooling and trapping mechanisms. We have measured the trapping beam intensity, detuning, magnetic-field gradient, trap density, and lifetime dependence of the spring constant $\kappa$ and damping coefficient $\alpha$ for a ${ }^{1} S_{0^{-}}{ }^{1} P_{1}{ }^{88} \mathrm{Sr}$ MOT by fitting the oscillatory response of the atom cloud to a step-function force. We find that the observed behavior of $\kappa$ and $\alpha$ provide a unified and consistent picture of trap dynamics that agrees with Doppler cooling theory at the level of $10 \%$. Additionally, we demonstrate that the trapped atom temperature can be determined directly from measured value of $\kappa$ and the trap size, in excellent agreement with free-expansion temperature measurements. However, the experimentally determined temperature is much higher than Doppler cooling theory, implying significant additional heating mechanisms.

DOI: 10.1103/PhysRevA.66.011401

PACS number(s): 32.80.Pj, 32.80.Lg, 39.25.+k, 42.50.Vk
\end{abstract}

Doppler cooling theory, which explores the force experienced by two-level atoms excited by single and/or multiple near-resonant light fields, has historically formed the basis for descriptions of both laser cooling and magneto-optical trapping (MOT) [1-6]. To date, however, experimental studies of laser cooling have focused nearly exclusively on systems such as alkali-metal atoms, wherein the transition's hyperfine structure ultimately dominates observed cooling and trapping dynamics [7-11]. While a one-dimensional (1D) study of Doppler cooling dynamics has been performed [12], fundamental Doppler cooling theory predictions, particularly those related to the dynamics of two-level atoms in a 3D MOT, remain untested.

In this paper, using a ${ }^{1} S_{0}{ }^{-1} P_{1}{ }^{88} \mathrm{Sr} \mathrm{MOT}$, we present measurements of the spring constant $\kappa$ and damping coefficient $\alpha$, as they are affected by the trapping beam intensity, detuning, magnetic-field gradient, trap density, and lifetime. We monitor directly the time-dependent center-of-mass trap oscillations induced by a chopped pushing beam. Unlike previous experiments with alkali-metal atoms [8-11], where sub-Doppler cooling mechanisms dominate over Doppler cooling, we observe center-of-mass cloud oscillations under both underdamped and overdamped conditions and find that the measured $\kappa$ and $\alpha$ are consistent with Doppler theory at the $10 \%$ level. Furthermore, we have determined the cloud temperature directly from the measured spring constant and trap size, just as in the case of magnetic traps. The results are in a good agreement with temperature measurements based upon free expansion of the cloud. However, both temperature measurements disagree significantly with Doppler theory, in showing a faster heating rate with the trapping beam intensity. Our measurements represent a detailed and conclusive study of trapped two-level atom dynamics in a MOT and an essential test of Doppler cooling theory.

The experiment consists of a standard six-beam vapor-cell Sr MOT using the ${ }^{1} S_{0^{-}}{ }^{1} P_{1}$ transition at $\lambda=461 \mathrm{~nm}$ (resonance frequency $\left.\omega_{0}\right)[13-15]$. The trapping light detuning,

\footnotetext{
*Email address: xyxu@jilau1.colorado.edu

${ }^{\dagger}$ Email address: Ye@jila.colorado.edu
}

$\left[\delta=\Delta /(2 \pi)=\left(\omega_{\text {laser }}-\omega_{0}\right) /(2 \pi)\right]$, is controlled by a double-passed acousto-optic modulator (AOM). The intensity of the trapping light is stabilized via feedback control of a second AOM.

To measure trap dynamics, we explored several different techniques including modulation of the magnetic field or a pushing beam. The following approach is finally adopted for this work due to its measurement efficiency and lack of systematic effects. Cold atom center-of-mass oscillation is induced with a weak, on-resonance, collimated pushing beam that is chopped with a switching time of $1 \mu \mathrm{s}$. The pushing beam exerts a force $F_{\text {ext }}$ on the atoms, translating the atom sample along one horizontal direction ( $x$ axis). The resulting trap oscillations are observed as changes in the absorption of an on-resonance probe beam that propagates in the $y$ direction through the cloud and is off-center in the $x$ direction. As shown below, the center-of-mass oscillation of the trapped atoms is described by

$$
m \frac{d^{2} x}{d t^{2}}+\alpha \frac{d x}{d t}+\kappa x=F_{e x t}
$$

where $m$ is the mass of a ${ }^{88} \mathrm{Sr}$ atom, $\alpha$ is a damping coefficient, and $\kappa$ is a spring constant. When the weak pushing beam is on, forced oscillations are observed, and $F_{\text {ext }}$ $\cong \frac{1}{2} \hbar k \Gamma\left(I_{p} / I_{s}\right)=F_{p}$. Here $k=2 \pi / \lambda, I_{p}$ is the pushing beam intensity, $I_{s}=\pi h c \Gamma /\left(3 \lambda^{3}\right)=43 \mathrm{~mW} / \mathrm{cm}^{2}$, and $\Gamma /(2 \pi)$ $=32 \mathrm{MHz}$ where $I_{s}$ and $\Gamma$ are the saturation intensity and natural width of ${ }^{1} S_{0^{-}}{ }^{1} P_{1}$ transition, respectively. When the pushing beam is switched off, $F_{\text {ext }}=0$, and free-oscillations occur. To quantify these dynamics, we define a dimensionless quantity $\xi=\alpha /(2 m \omega)$, where $\omega=\sqrt{\kappa / m}$ is the characteristic frequency of the trap. Underdamped, critically damped, and overdamped oscillations correspond to $\xi<1$, $\xi=1$, and $\xi>1$, respectively. Denoting $u_{t}$ as the normalized trap displacement during oscillation, the steady-state solutions to Eq. (1) in response to a step-function force take the following form for $F_{\text {ext }}=0$ : 


$$
\begin{gathered}
u_{t}(\xi<1)=\frac{e^{-\xi \omega t}}{\sqrt{1-\xi^{2}}} \sin \left[\omega \sqrt{1-\xi^{2}} t+\tan ^{-1}\left(\frac{\sqrt{1-\xi^{2}}}{\xi}\right)\right] \\
u_{t}(\xi=1)=(1+\omega t) e^{-\omega t}, \\
u_{t}(\xi>1)=\frac{1}{p_{1}-p_{2}}\left[-p_{2} e^{p_{1} t}+p_{1} e^{p_{2} t}\right],
\end{gathered}
$$

where $u_{t}$ is normalized to the $t \rightarrow \infty$ displacement of the trap and $p_{1,2}=-\xi \omega \pm \omega \sqrt{\xi^{2}-1}$. When $F_{\text {ext }}=F_{p}$, the steadystate solutions to Eq. (1) are $1-u_{t}$, where $u_{t}$ is given by Eqs. (2), (3), and (4).

Equation (1) and its solutions, Eqs. (2)-(4), describe the motion of an atom initially at rest off center in a harmonic potential, oscillating as it comes to rest at the center of this potential. Here we verify that this situation describes the center-of-mass motion of the entire cloud of finite temperature atoms, and that the cloud shape does not vary during oscillation. The 1D Focker-Planck equation, which includes heating due to the momentum diffusion and describes the phase-space distribution of the entire atom cloud [5], is

$$
\frac{\partial P}{\partial t}=a \frac{\partial P}{\partial v}-v \frac{\partial P}{\partial x}+\frac{\partial}{\partial v}\left(\frac{\alpha v}{m} P+D \frac{\partial P}{\partial v}\right) .
$$

Equation (5) governs the motion of the ensemble of the trapped atoms, where $v=d x / d t, a=d^{2} x / d t^{2}=F_{\text {ext }} / m$, due to the applied conservative forces in the trap, $D$ $=\hbar^{2} k^{2} \Gamma I_{t} /\left[6 m^{2} I_{s}\left(1+I_{t} / I_{s}+4 \Delta^{2} / \Gamma^{2}\right)\right]$ is the velocity diffusion coefficient, and $I_{t}$ is the total intensity of the six trapping beams. The steady-state solution to Eq. (5) is

$$
P(x, v)=\exp \left[-A\left(v^{2}+\frac{\kappa}{m} x^{2}\right)\right]
$$

where $A=\alpha /(2 D m)$. Equation (6) also gives the canonical distribution at temperature $k_{B} T=m^{2} D / \alpha$. Based on Doppler cooling theory, the one-dimensional spring constant and damping coefficient can be described by $[4-6,14]$,

$$
\begin{gathered}
\kappa=\frac{4}{3} \mu_{B} g_{J} \frac{\partial B}{\partial x}\left(\frac{|\Delta|}{\Gamma}\right)\left(\frac{I_{t}}{I_{s}}\right)\left(1+\frac{I_{t}}{I_{s}}+4 \frac{\Delta^{2}}{\Gamma^{2}}\right)^{-2}, \\
\alpha=\frac{4}{3} \hbar k^{-2}\left(\frac{|\Delta|}{\Gamma}\right)\left(\frac{I_{t}}{I_{s}}\right)\left(1+\frac{I_{t}}{I_{s}}+4 \frac{\Delta^{2}}{\Gamma^{2}}\right)^{-2},
\end{gathered}
$$

where the relevant magnetic-field gradient is $\partial B / \partial x$ $=0.5 \partial B / \partial z$, with $\partial B / \partial x(\partial B / \partial z)$ being the radial (axial) MOT magnetic-field gradients. The solution of Eq. (5) subject to the initial conditions of a Gaussian distribution of velocity and position with a displaced center is

$$
P(x, v, t)=\exp \left(-A\left[\left(v-u_{t}^{\prime}\right)^{2}+\frac{\kappa}{m}\left(x-u_{t}\right)^{2}\right]\right)
$$
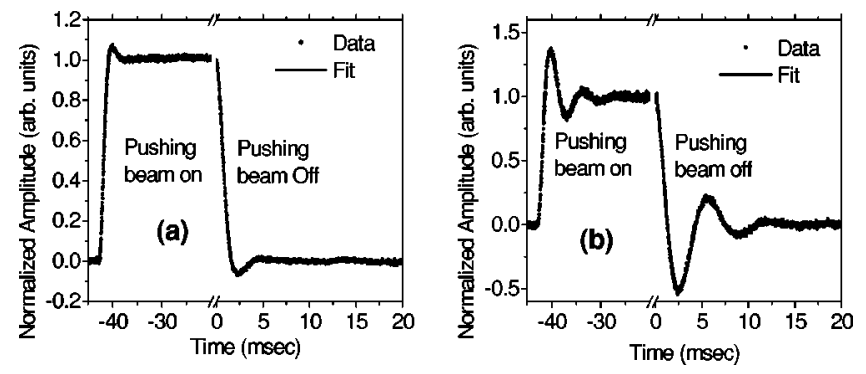

FIG. 1. Typical damped oscillation signals for trapped ${ }^{88} \mathrm{Sr}$ atoms during a pushing beam chopping cycle for two different detunings, (a) $\delta=-40 \mathrm{MHz}$ and (b) $\delta=-55 \mathrm{MHz}$. Note the time scale is expanded by a factor of 2 after the break. Fitting curves are shown as solid lines. The intensity of the trapping beam is 25 $\mathrm{mW} / \mathrm{cm}^{2}$, and $\partial B / \partial x=26 \mathrm{G} / \mathrm{cm}$.

where $u_{t}$ is the solution to Eq. (1) and $u_{t}^{\prime}=d u_{t} / d t$. Thus at all times the velocity distribution is centered on $u_{t}^{\prime}$ and the spatial distribution is centered on $u_{t}$, with a constant size and temperature.

In the experiment, the cloud's displacement is monitored by a weak $100 \mu \mathrm{m}$-diameter (1/e) on-resonance focused probe beam that propagates perpendicular to the pushing beam as it passes through the atom cloud. The probe beam is located at $x_{0}$, which is about half a cloud radius from the cloud center in the $x$ direction at $z=0$. Thus, the normalized probe beam absorption is $S_{a b s}=1-\exp \left[-\sqrt{\pi} \sigma n(x, t) r_{h}\right]$, where $\sigma=3 \lambda^{2} /(2 \pi)$ is the absorption cross section, $n(x, t)$ $=n_{0} e^{-\left(x_{0}+x_{m} u_{t}\right)^{2} / r_{h}^{2}}$, where $n_{0}$ is the peak trap density, $r_{h}$ is the 1/e radius of the trap along the $x$ and $y$ axis, and $x_{m}$ is the atom cloud displacement when the pushing beam is on. The absorption is $<10 \%$ for a typical trap density of $10^{9} / \mathrm{cm}^{3}$. If $x_{m} / r_{h} \ll 1$, the absorption signal is given by

$$
S_{a b s}=S_{a b s}\left(x_{0}\right)\left[1-\epsilon u_{t}\right] \text {, }
$$

where $S_{a b s}\left(x_{0}\right)$ is the probe beam absorption at $x_{0}$ with pushing beam off and $\epsilon=2 x_{0} x_{m} / r_{h}^{2}$. Equation (10) shows the absorption is proportional to $u_{t}$ when the amplitude $x_{m}$ is much smaller than the trap size. Hence, the time-dependent probe beam absorption represents center-of-mass trap oscillations.

Figure 1 shows oscillation signals for $I_{t}=25 \mathrm{~mW} / \mathrm{cm}^{2}$, $\partial B / \partial x=26 \mathrm{G} / \mathrm{cm}$, and laser detunings of (a) $\delta=-40 \mathrm{MHz}$ and (b) $\delta=-55 \mathrm{MHz}$. In the absence of the pushing beam, this trap oscillation is driven purely by the trap fields. Note that, from Eq. (2), the number of observable oscillations is proportional to $\xi$. Equations (7) and (8) thus predict that large detunings or low intensities correspond to a large number of observable oscillations. In both cases the trap oscillations are underdamped, which is quite different from alkali MOTs where the oscillation is strongly overdamped due to sub-Doppler cooling mechanisms [8-11].

Experimentally, observation of high-contrast oscillation signals requires: (1) Balancing the six-beam intensities and aligning the trapping beams such that the cloud does not move when the trap intensity changes; (2) Ensuring the probe beam intersects the cloud midway between the center 

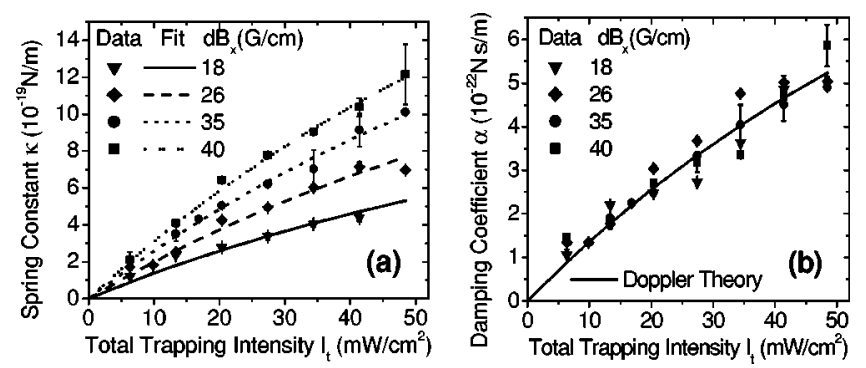

FIG. 2. Dependence of (a) $\kappa$ and (b) $\alpha$ on the trapping beam intensity for various magnetic-field gradients and a trapping light detuning of $\delta=-40 \mathrm{MHz}$. Fits are shown as solid and dotted lines in (a). The solid line in (b) is a theoretical curve based on Doppler theory.

and edge; (3) Aligning the push beam such that it induces motion only in the horizontal plane; (4) Attenuating the pushing beam such that the maximum cloud displacement is $<10 \%$ of the cloud diameter, with no noticeable heating effects. We find the oscillation signals are independent of probe beam placement on either side of the cloud. The values of $\kappa$ and $\alpha$ are determined by fitting of the free-oscillation signals, as shown with the solid lines in Fig. 1.

Figure 2 shows the dependence of (a) $\kappa$ and (b) $\alpha$ on the trapping beam intensity, $I_{t}$, for magnetic-field gradients, $\partial B / \partial x$, ranging from $18-42 \mathrm{G} / \mathrm{cm}$ and a trap detuning $\delta=$ $-40 \mathrm{MHz}$. For comparison, Fig. 2(a) shows fitting curves based on Eq. (7) while Fig. 2(b) shows theory predictions based on Eq. (8). For these experimental conditions, Eq. (7) predicts $\kappa=\eta_{\kappa} i_{\text {coil }} I_{t} /\left[I_{s}\left(7.25+I_{t} / I_{s}\right)^{2}\right]$, where $i_{\text {coil }}$ is the quadrupole magnetic-field coil current and $\partial B / \partial x=\beta_{x} i_{\text {coil }}$. Using a best estimated value of $\beta_{x}=4.4(0.5) \mathrm{G} \mathrm{cm}^{-1} \mathrm{~A}^{-1}$, the theoretical value for $\eta_{\kappa}$ is 92.0(18.4) $\times 10^{-9} \mathrm{Nm}^{-1} \mathrm{~A}^{-1}$ where the uncertainty in $\eta_{\kappa}$ is dominated by uncertainties in $\beta_{x}, I_{t}$, and $\delta$. Fitting the data to this expression with $\eta_{\kappa}$ as the only fitting parameter yields $\eta_{\kappa}=83(3), 80(2), 77(1)$, and $83(1) \times 10^{-19} \mathrm{Nm}^{-1} \mathrm{~A}^{-1}$ for $i_{\text {coil }}=4,6,8$, and $9 \mathrm{~A}$, or $\partial B / \partial x=18,26,35$, and $40 \mathrm{G} / \mathrm{m}$, respectively. Here, the nearly equal values for $\eta_{\kappa}$ show that $\kappa$ is proportional to the magnetic-field gradient, and the variation with $I_{t}$ shows the dependence expected from Eq. (7). These coefficients are consistent with Doppler cooling theory predictions within experimental accuracy of $20 \%$. For the conditions of Fig. 2(b), Eq. (8) yields $\alpha=\eta_{\alpha} I_{t} /\left[I_{s}(7.25\right.$ $\left.\left.+I_{t} / I_{s}\right)^{2}\right]$ with $\eta_{\alpha}=326(65) \times 10^{-22} \mathrm{~N} \mathrm{~s} / \mathrm{m}$. Using $\eta_{\alpha}$ as a single fitting parameter we find $\eta_{\alpha}=315(16), 349(12)$, $318(5)$, and $331(16) \times 10^{-22} \mathrm{~N} \mathrm{~s} / \mathrm{m}$ for $i_{\text {coil }}=4,6,8$, and 9 A, respectively. The nearly equal values for $\eta_{\alpha}$ demonstrate that $\alpha$ is independent of $\partial B / \partial x$, while the variation with $I_{t}$ shows the expected dependence from Eq. (8). The measured values for $\eta_{\alpha}$ agree with Doppler cooling theory predictions within experimental uncertainty of $10 \%$.

Figure 3 shows the detuning dependence of (a) $\kappa$ and (b) $\alpha$ for $I_{t}=25 \mathrm{~mW} / \mathrm{cm}^{2}$ and $\partial B / \partial x=26 \mathrm{G} / \mathrm{cm}$. Fitting curves based on Eqs. (7) and (8) are shown as solid lines. Here, Eqs. (7) and (8) predict $\kappa=\chi_{\kappa}|\Delta| /\left[\Gamma\left(1.58+4 \Delta^{2} / \Gamma^{2}\right)^{2}\right]$, and $\alpha$ $=\chi_{\alpha}|\Delta| /\left[\Gamma\left(1.58+4 \Delta^{2} / \Gamma^{2}\right)^{2}\right]$, where theoretical values of $\chi_{\kappa}$ and $\chi_{\alpha}$ are $259(52) \times 10^{-19} \mathrm{~N} / \mathrm{m}$ and $152(30) \times 10^{-22}$
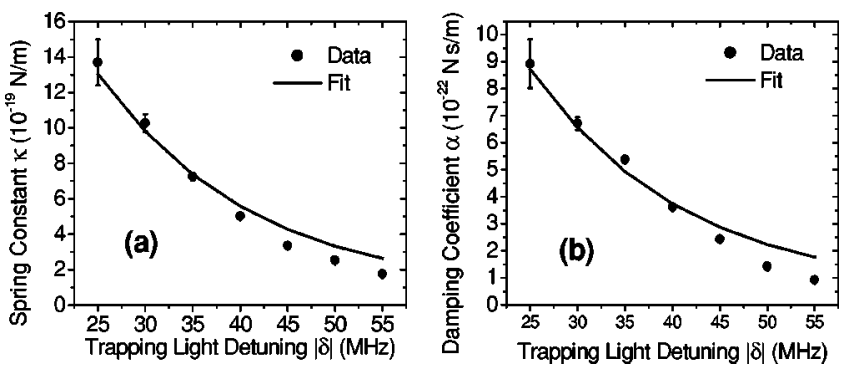

FIG. 3. Dependence of (a) $\kappa$ and (b) $\alpha$ on the trapping light detuning for a trapping beam intensity of $25 \mathrm{~mW} / \mathrm{cm}^{2}$ and $\partial B / \partial x$ $=26 \mathrm{G} / \mathrm{cm}$. Fits are shown as solid lines.

$\mathrm{N} \mathrm{s} / \mathrm{m}$, respectively. From fits to the data, we find $\chi_{\kappa}$ $=272(10) \times 10^{-19} \mathrm{~N} / \mathrm{m}$ and $\chi_{\alpha}=182(7) \times 10^{-22} \mathrm{~N} \mathrm{~s} / \mathrm{m}$, in good agreement with the theoretical predictions. Both $\kappa$ and $\alpha$ increase rapidly with decreasing detuning, in agreement with theory at small detunings. However, so far there is no clear explanation for the origin of the discrepancy between the measured and fitted values at large detunings.

Based on the equipartition theorem, $\frac{1}{2} \kappa x_{r m s}^{2}=\frac{1}{2} k_{B} T$, we can determine the cloud temperature from the measured spring constant and trap size. To determine the trap size, we use images of trap fluorescence collected with a charge coupled device camera placed in the $y$ direction. The profile of the cloud is well fitted by a Gaussian distribution. The 1/e radius of the cloud along the oscillation direction is defined as $x_{e}=\sqrt{2} x_{r m s}$; hence, the trap temperature is given by $T$ $=\kappa x_{e}^{2} /\left(2 k_{B}\right)$. The dependence of the trap temperature on the trapping beam intensity for the data in Fig. 2(a) is shown as Fig. 4, along with temperatures predicted by Doppler theory [6],

$$
T=\frac{\hbar \Gamma^{2}}{8 k_{B}|\Delta|}\left(1+\frac{I_{t}}{I_{s}}+4 \frac{\Delta^{2}}{\Gamma^{2}}\right) .
$$

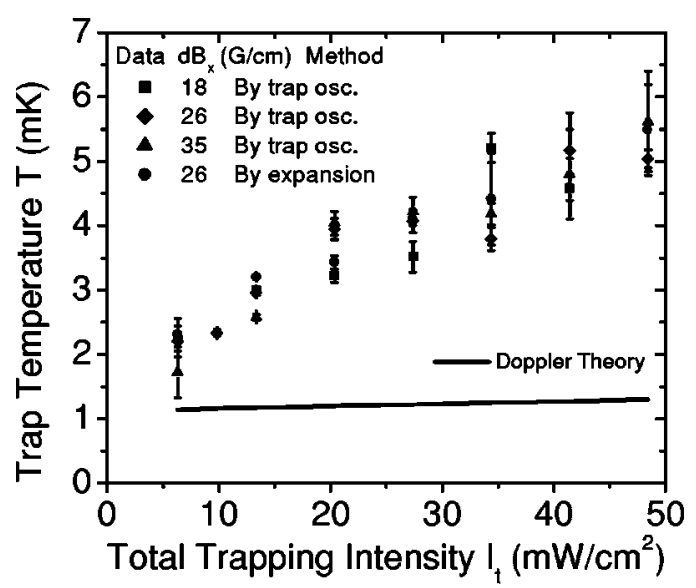

FIG. 4. Dependence of the trap temperature on the trapping beam intensity as determined from the measured $\kappa$ values. The theoretical prediction is shown as a solid line. The trap temperature, determined from the expansion measurement for $\delta=-40 \mathrm{MHz}$ and $\partial B / \partial x=26 \mathrm{G} / \mathrm{cm}$, is shown as filled circles. 
The measured trap temperature is independent of the magnetic-field gradient, in agreement with theoretical predictions. However, we observe important differences: while the trap temperature at near zero intensity is correctly predicted by Doppler theory, the temperature rises with the trapping beam intensity much faster than the theoretical values. Our measured temperatures agree with previous measurements in this Sr MOT based on direct observation of the cold atom velocity distribution [14]. Similar temperature deviations from Doopler theory have also been observed for a Ca MOT [16]. We have also found that the dependence of the trap temperature on the trapping beam detuning is significantly different from Doppler theory. For example, at $\Delta / \Gamma \sim 1.2$, the measured temperature is about three times higher than predicted. At smaller detunings the disagreement becomes progressively larger.

To further verify these results, we have measured the trap temperature vs $I_{t}$ for $\delta=-40 \mathrm{MHz}$ and $\partial B / \partial x=26 \mathrm{G} / \mathrm{cm}$ with a conventional expansion technique. For these measurements, we shifted the probe beam to the center of the cloud and observed the time dependence of the probe beam absorption after the MOT is turned off. The resulting decay curves were then fit by $f(t)=\zeta_{1} e^{-\zeta_{2} /\left(r_{o}^{2}+2 v_{r m s}^{2} t^{2}\right)}$, where $\zeta_{1}, \zeta_{2}$, $v_{r m s}$ are three fitting parameters, and $r_{0}=\sqrt[3]{r_{e x} r_{e y} r_{e z}}, r_{e x}$, $r_{e y}$, and $r_{e z}$ are the 1/e radii of the cloud in three dimensions as determined from fits to images of the 461-nm trap fluorescence. Using the fitting parameter $v_{r m s}$, we obtain the trap temperature from $T=m v_{r m s}^{2} / k_{B}$. Results are shown as filled circles in Fig. 4, and agree with values determined from the spring constant at the level of $10 \%$.

The trapped atom temperature is determined by a dynamic balance between heating and cooling rates. The damping coefficient represents the cooling rate, and as shown in Figs. 2 and 3, measured values for the damping coefficients agree well with Doppler cooling theory. However, the measured temperatures are much higher than the theoretical values, so the heating rate must be much higher than the theoretical prediction, which is based on random direction photon recoil heating [the $D$ term in Eq. (5)]. Additional heating mechanisms, such as that arising from standing-wave effects, are not described by current Doppler theory.

The loss rate for a $\mathrm{Sr}^{1} S_{0^{-}}{ }^{1} P_{1}$ MOT is $\sim 10^{2} / \mathrm{s}$ due to $5 p^{1} P_{1} \rightarrow 4 d^{1} D_{2} \rightarrow 5 p^{3} P_{2}$ shelving. To verify that this loss mechanism does not significantly influence trap oscillation dynamics, we also measured the spring constant and damping coefficient in the presence of two repumping lasers, one for the ${ }^{3} S_{1}-{ }^{3} P_{2}$ transition at $707 \mathrm{~nm}$ and a second for the ${ }^{3} S_{1}-{ }^{3} P_{0}$ transition at $679 \mathrm{~nm}$, in order to prevent ${ }^{3} P$ state shelving. Even though the density and lifetime of the trap were both increased by more than six times, the measured values for $\kappa$ and $\alpha$ did not change within our experimental uncertainty of $10 \%$. This means that the spring constant and damping coefficient are independent of the density and the trap lifetime for densities of $10^{8}-10^{10} \mathrm{~cm}^{-3}$ and lifetimes of $10-400 \mathrm{~ms}$.

In summary, we have performed the measurement of the spring constant $\kappa$ and damping coefficient $\alpha$ for two-level atoms in a MOT. Our results for $\kappa$ and $\alpha$ as functions of laser intensity and detuning, and of magnetic-field gradient are in agreement with the Doppler cooling theory. In addition, we have measured the trap temperature by using two methods, first from the spring constant and trap size, and second from expansion of the cloud after switching off the MOT. The temperatures measured by the two techniques agree well with each other, but deviate severely from Doppler theory. At present, a modified semiclassical Doppler theory is being developed to investigate heating effects that are not accounted for by standard Doppler theory. In future experiments, we will further study the dynamics of the trapped atoms after second-stage Doppler cooling, using the spectrally narrow 689-nm ${ }^{1} S_{0^{-}}{ }^{3} P_{1}$ intercombination line $[14,15]$. Since the $689-\mathrm{nm}$ photon recoil frequency shift is greater than the cooling transition linewidth, a full quantummechanical cooling theory will be necessary.

We thank Dr. J. R. Bochinski for useful discussions. This work was funded by NSF and NIST. T.H.L. acknowledges financial support from the National Research Council.
[1] T.W. Hänsch and A.L. Schawlow, Opt. Commun. 13, 68 (1975).

[2] D. Wineland and H. Dehmelt, Bull. Am. Phys. Soc. 20, 637 (1975).

[3] A. Ashkin, Science 210, 1081 (1980).

[4] V.S. Letokhov and V.G. Minogin, Phys. Rep. 73, 1 (1981).

[5] S. Stenholm, Rev. Mod. Phys. 58, 699 (1986).

[6] P.D. Lett, W.D. Phillips, S.L. Rolston, C.E. Tanner, R.N. Watts, and C.I. Westbrook, J. Opt. Soc. Am. B 6, 2084 (1989).

[7] E.L. Raab, M. Prentiss, A. Cable, S. Chu, and D.E. Pritchard, Phys. Rev. Lett. 59, 2631 (1987).

[8] A.M. Steane and C.J. Foot, Europhys. Lett. 14, 231 (1991).

[9] P. Kohns, P. Buch, W. Suptitz, C. Csambal, and W. Ertmer, Europhys. Lett. 22, 517 (1993).

[10] A. Hope, D. Haubrich, G. Muller, W.G. Kaenders, and D. Me- schede, Europhys. Lett. 22, 669 (1993).

[11] C.D. Wallace, T.P. Dinneen, K.Y.N. Tan, A. Kumarakrishnan, P.L. Gould, and J. Javanainen, J. Opt. Soc. Am. B 11, 703 (1994).

[12] D. S. Weiss, E. Riis, Y. Shevy, P.J. Ungar, and S. Chu, J. Opt. Soc. Am. B 6, 2072 (1989).

[13] T.P. Dinneen, K.R. Vogel, E. Arimondo, J.L. Hall, and A. Gallagher, Phys. Rev. A 59, 1216 (1999).

[14] K. Vogel Ph.D. thesis, University of Colorado, Boulder, 146. (1999).

[15] H. Katori, T. Ido, Y. Isoya, and M. Kuwata-Gonokami, Phys. Rev. Lett. 82, 1116 (1999).

[16] Th. Kisters, K. Zeiske, F. Riehle, and J. Helmcke, Appl. Phys. B: Photophys. Laser Chem. 57, 89 (1994). 